\title{
Algorithm Of Eye Location With Gray Projection And Hough Transform Based On ARM Platform
}

\author{
Caixia Liu \\ Department of information science and engineering, Zaozhuang University, Zaozhuang, 277160. \\ China
}

Keywords: ARM, gray-level projection, adaptive threshold, Hough transform

\begin{abstract}
Image processing system based on ARM is a faster, smaller and more flexible application. Based on the analysis of human face and eye features, a new eye location algorithm based on ARM embedded system is presented. Firstly, the methods of histogram equalization and median filtering are used for the image enhancement and noise reduction. The adaptive Niblack method is used for segmentation and binarization. The approximate location of eyes is gotten by the horizontal and vertical gray-level histogram. Finally, on the basis of the analysis of the grayscale characteristics of human eye, Hough transform is used to get the right eye position. Experimental results show that this algorithm is easy to realize, reduces the blindness of the search process, and improves the speed.
\end{abstract}

\section{Introduction}

Eye automatic positioning is a basic and very important Subject research in the study of face recognition. Compared with facial parts such as nose and mouth, eyes area contains more important information which can be used to distinguish individual. At present, the most commonly eye location method is the deformable template method [1] proposed by Yuille, etc. Its advantage is that it can get the location and shape information of the eye at the same time, the disadvantage is the slow speed. What is more, the convergence of optimization of process is often depends on the choice of initial parameters values. The other eye location methods are: Hough transform method [2], the generalized symmetry transform method, projection curve method, template matching method [3], principal component analysis method [4], etc. The former two methods need large amount of calculation, and the last two methods need higher image quality. In addition, the neural network method on eye location often needs a large number of training samples. If there are not a lot of samples fully considering rotating angle, scale and illumination changes, it is difficult to use this method.

There are many kinds of image segmentation methods and can be divided into two main categories. One is boundary method. The other is area method. In image segmentation methods, the threshold is the most simple and effective method. It is a kind of traditional image segmentation method and has become the most widely used technology in image segmentation because of its small amount of calculation stable performance and easy to implement.

The key is to find the proper threshold on threshold image segmentation, The threshold is used to distinguish the image object and background. When gray difference of the image objects and the background is large, it is easy to find a global threshold of image segmentation and can obtain satisfactory results. When the image is more complex and image gray level change is bigger, the local details of the image will be ignored with image segmentation with global threshold. The dynamic threshold segmentation is considered to improve the image segmentation effect. Niblack threshold segmentation method is a kind of classic threshold segmentation algorithm, but the choice of the correction coefficient is random.

With the development of embedded microcontroller technology, digital signal processing theory is gradually applied to the actual system.In this paper, the eye location method based on gray projection and haugh transformation on ARM embedded systme is proposed. First, the image preprocessing is used to separate face and background, remove the noise. Adaptive Niblack threshold segmentation method is used for image binarization. Vertical gray-level histogram is used to segment face, narrow 
eyes monitoring area, at the same time positioning the eyes vertical area. Because the person's two eyes are not necessarily in a horizontal line, the left and right horizontal projections are used to locate the position of the two eyes. The approximate locations of the two eyes are determined according to the above methods. Finally through the analysis of the characteristics of the eye, the approximate location of the iris are collected, the exact location of eyes are located with fast hough transform.

\section{Hardware Platform}

Image processing functions are applied on the system with ARM920T core and some peripheral circuits. A variety of communication interfaces make the image transmission channel and the image data is extracted with SD card interface, as shown in Figure 1. Here the choice LCD is LTV350QV-F05 of 3.5-inch. Image data processing module uses Samsung's S3C2410A.

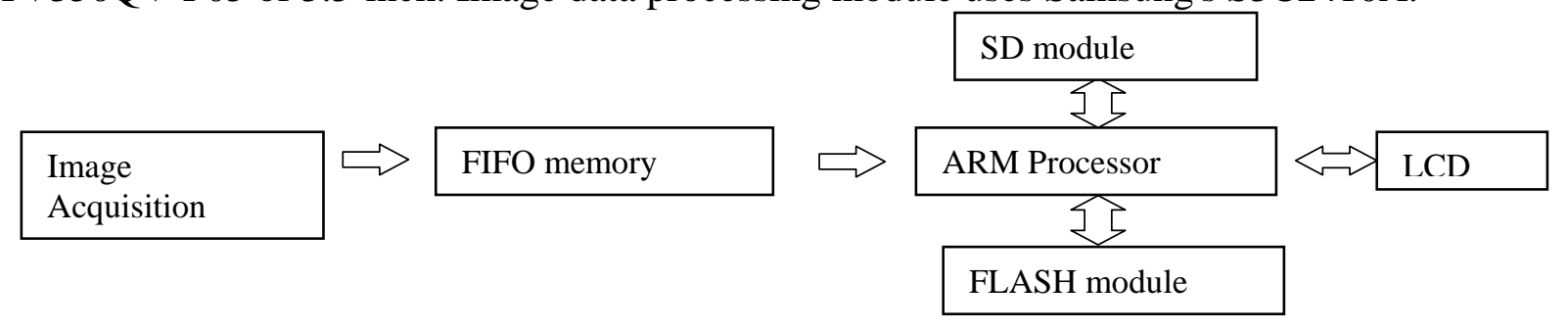

Fig. 1 Hardware platform

At the time of detection, images collected by image acquisition system will be stored in FIFo memory, ARM acquires the image data from FIFO module and processes image.

\section{Algorithm of eye Location Based on Gray Projection and Hough Transform}

Histogram Equalization. The background is removed first with the following formula before histogram equalization.

$$
\mathrm{A}(x, y)=\left\{\begin{array}{l}
0, \text { where } I(x, y)<\text { mean }(I) \\
I(x, y), \text { otherwise }
\end{array}\right.
$$

Where I $(x, y)$ is the input image, mean $(I)$ is image mean and $\mathrm{A}(x, y)$ is the result.

In general, image transmission and transformation (such as imaging scanning transmission and display, etc.) in all kinds of image system will cause certain reduction. For example, the optical system distortion, relative movement, air flow when capturing the image can make the image fuzzy. During transmission, the image quality will be declined due to the noise pollution. The image enhancement processing is needed. Histogram equalization algorithm is a simple and efficient enhancement method.

Smoothing filter based on space and frequency domain can suppress image noise, improve signal-to-noise ratio of image. In order to remove the noise in the image, many methods are proposed. Among them, the median filtering is a local smoothing technique [1]. It has very good effect on pulse interference and salt and pepper noise. Figure 2 (a) is the original image, (b) is the histogram of the original image.

Adaptive Niblack Method. The selection of image segmentation threshold is the key technology. Whether the selection is reasonable will directly affect the segmentation result and the subsequent processing of the image. In order to get more accurate and reliable threshold, many scholars have conducted extensive and in-depth research [5].

Niblack method is a simple and effective local dynamic threshold algorithm. The basic idea of the this algorithm is to calculate the mean and variance of pixels in neighborhood in its $r \times r$ neighborhood, and then use formula (2)to calculate the binary value.

$$
\mathrm{T}(x, y)=\mathrm{m}(x, y)+k^{*} s(x, y)
$$




$$
\begin{aligned}
& m(x, y)=\frac{1}{r^{2}} \sum_{i=x-r / 2}^{x+r / 2} \sum_{j=y-r / 2}^{y+r / 2} f(i, j) \\
& s(x, y)=\sqrt{\frac{1}{r^{2}} \sum_{i=x-r / 2}^{x+r / 2} \sum_{j=y-r / 2}^{y+r / 2} f(i, j)^{2}}
\end{aligned}
$$

Where, for each pixel point $(x, y), \mathrm{T}(x, y)$ is the threshold of this point, $m(x, y)$ is the average of $r \times$ $r$ neighborhood, $\mathrm{s}(x, y)$ is the standard variance. $k$ is the correction coefficient.

The correction coefficient k generally depends on experience, and is constant for the whole image. So there is certain randomness. If choose is not appropriate, it will be hard to get effective results. The result is good when the $\mathrm{k}$ is 0.2 as shown in figure3.

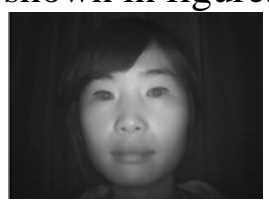

(a)

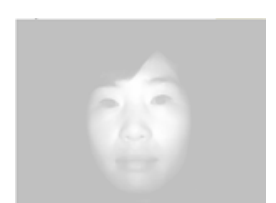

(b)

Fig.2 Histogram equalization. (a)original image; (b)the result.

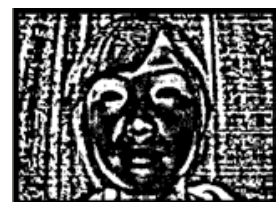

(a)

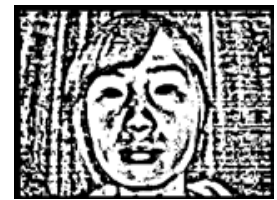

(b)

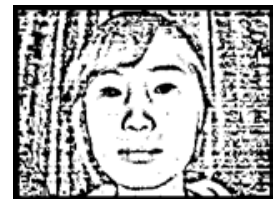

(c)

Fig.3Segmentation results under different threshold of original Niblack (a)k=0.2; (b)k=0; (c)k=-0.2.

The selection of $k$ effect the result, but the selection of $k$ is random. Here, we calculate $\mathrm{k}$ in every window.

$$
\begin{aligned}
& k w=m(x, y) /(s(x, y)+\varepsilon) \\
& k=\left\{\begin{array}{lc}
-\log (k w)^{*} 0.1 & k w>1 \\
\log (k w) & k w<=1
\end{array}\right.
\end{aligned}
$$

Where, $\varepsilon$ is a little value and can be 1 in order to avoid the denominator is zero in the process of operation. $\mathrm{k}$ is obtained by formula (8). The value of $\mathrm{k}$ is different with the mean and standard deviation in different window. The segmentation results will be more accurate.

Figure 4 shows the experimental results with different methods including the algorithm proposed in this paper. Figure 4(a)-(c) is the results with other segmentation algorithm. We can see from figure 4 , the segmentation results have less noise regardless of the size of the template, and the segmentation results is better. By contrast, our algorithm has more details as shown in figure4(a).

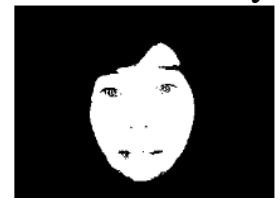

(a)

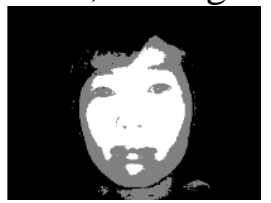

(b)

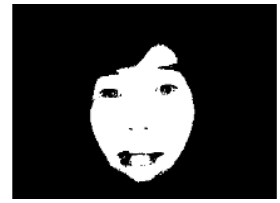

(c)

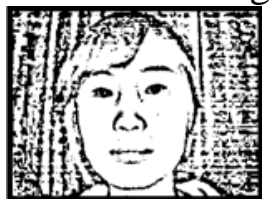

(d)

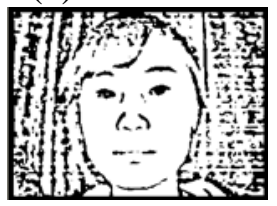

(e)

Fig.4 Image segmentation. (a) OTSU method(N=2); (b) K-means clustering method(N=3); (c) global thresholding method ( $\mathrm{t}=100)$; (d)our method;(e)result with median filter.

Combining with adaptive Niblack threshold, binarization and background separation is calculated and figure 4(d) is get.

Gray Projection. Find horizontal gray-level histogram of figure 5(b), as shown in figure 5(c).The first minimum value is the location of the eyebrows from the left side, the second minimum point is 
eye location. Assuming the eye is at horizontal line $\mathrm{K}$, with $\mathrm{K}+25, \mathrm{~K}-15$ defining two eyes location, and make level segmentation. Make vertical histogram statistics of figure 5 (b) and get figure5 (d). Combined with twice projection[6], looking for two minimum points and can be taken as eye location as shown in figure 5 (f).

Gray projection method is simple, but it is limited to the front face image, namely, there are two eyes. Also, if two eyes is not in a level line, this method has limitations.

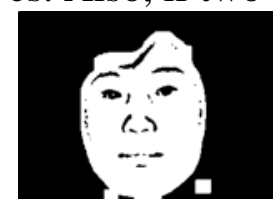

(a)

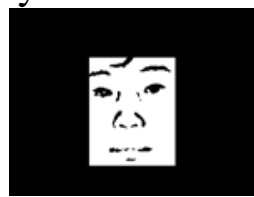

(b)

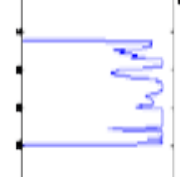

(c)

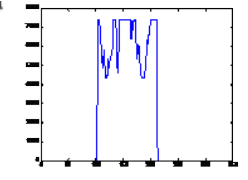

(d)

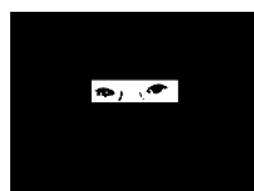

(e)

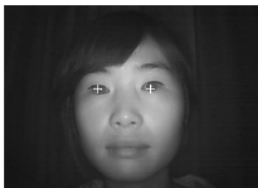

(f)

Fig.5 (a) binarization; (b) background segmentation; (c) a horizontal gray-level projection; (d) vertical gray-level projection; (e) segmentation result; (f) initial human eye localization.

Hough Transform.Hough Transform is a kind of feature extraction in image processing technology, it detected the shape of the object by a vote way. Hough Transform gets a collection of the specific shape as a Hough transform result by calculating the local maximum of cumulative results in a parameter space. Hough transform is put forward by Paul Hough [7] in 1962 and promote the use in 1972 by Richard Duda Peter Hart. Classical Hough transform is used to detect the straight line of image, then Hough transform extended to arbitrary shape object recognition, most of them are circles and ellipses.

At the initial projection position, find the general location of pupil circle center. Based on the detection of pupil, the position of the eyes can be detected by Hough transform.

\section{Analysis of experimental results}

The energy image was gotten by Haugh transformation on the face position of original image as shown in figure 6(a). Combined with the horizontal and vertical position of eye initial position, the two point with largest energy was found and can be taken as the eye position as shown in figure 6(b).

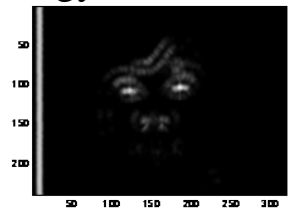

(a)

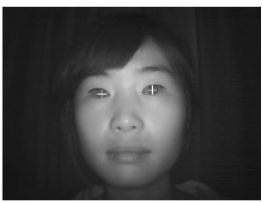

(b)

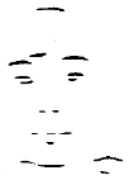

(c)

Fig. 6 (a) Haugh transformation; (b) eye location; (c) the method of literature[8].

For example, horizontal filtering was directly imposed on the original image as shown in figure 6 . Eyes, eyebrows and mouth can be clearly detected. That is to say, this method can be use after the face position was detected. However, this method needs large amount of calculation.

\section{Conclusions}

A new algorithm of eye location method with gray projection and Haugh transformation based on ARM platform is proposed. Combined with histogram equalization, adaptive Niblack threshold segmentation method, and gray-level projection, the position of eyes are initially located. Because the person's two eyes are not necessarily in a horizontal line, the exact location of eyes are located with hough transform based on the initial location.

\section{References}

[1] A L Yuille J.Handbook of Research on Face Processing,Cognitive Neuroscience,vol.3,1989,pp. 59-70. 
[2] Wang Lei, Mo Yu Long. Automatic eye feature extraction based on Hough transform and eyelid deformable template, Journal of Infrared and Millimeter Waves,vol.18(1999),No.1, p.53-60.

[3] Brunelli R,Poggio.T. Face recognition Features versus templates, IEEE Transactions on Pattern Analysis and Machine Intelligence,vol.15(1993), No.103,p. 1042-1052.

[4] Pentland A,Moghanddam B,Stamer T. View based and modular Eigenspaces for face recognition, Proceedings of IEEE Conference on Computer Vision and Pattern Recognition Seattle, 1994,No.1,p. 84-91.

[5] Wang Hai-Yang Pan De-Lu Xia De-Shen. A Fast Algorithm for Two-dimensional Otsu Adaptive Threshold Algorithm, Acta Automatica Sinica,vol.33(2007), No.9,p.968-971.

[6] Zhang Nana, Ma Yan, Su Guilian. Method of Eye Location Based on Gray Projection Function, Computer Engineering,vol.32(2006), No.10,p.193-195.

[7] Hough P V C. Method and means of recognizing complex patterns. U.S.Patent 3069654,1962

[8] Xiong Fei. Method of Eye Location Based on Gabor filter, Computer \& Digital Engineering,vol.35(2007), No.11,p.16-18. 